\title{
Fourth-line rescue therapy with rifabutin for $H$. pylori infection
}

In a small number of patients with Helicobacter pylori infection, three rounds of treatment do not achieve eradication. A Spanish Study Group has recently shown that a fourth-line therapy containing rifabutin has some success in these patients.

Rifabutin has shown promise in experimental conditions. "Consequently, rifabutin-based rescue therapies represent a potential strategy for eradication failures," explains corresponding author Javier Gisbert. "However, the experience with this drug in H. pylori treatment is still very limited."

The study included 100 patients who had previously not responded to three treatments. They were given $150 \mathrm{mg}$ rifabutin, $1 \mathrm{~g}$ amoxicillin and $\mathrm{PPI}$ at the standard dose twice daily for 10 days. Eradication was confirmed with a ${ }^{13} \mathrm{C}$-urea breath test 4-8 weeks after therapy. The perprotocol and intention-to-treat eradication rates were $52 \%$ and $50 \%$, respectively. 30 of the patients reported adverse events, such as nausea/vomiting and abdominal pain.
The authors note that their study seems to be the largest so far to evaluate rifabutin as a treatment for $H$. pylori infection, particularly in patients who have previously not responded to three or more treatments.

These findings indicate that a rescue therapy containing rifabutin is a useful strategy in patients who have not responded to treatment with the major antibiotics (such as amoxicillin, clarithromycin, metronidazole, tetracycline and levofloxacin). "However, we still need to find the ideal therapeutic strategy for the $50 \%$ of patients who do not respond to rifabutin-based treatment, and that is our goal for the near future," concludes Gisbert.

Claire Greenhill

Original article Gisbert, J. P. et al. Fourth-line rescue therapy with rifabutin in patients with three Helicobacter pylori eradication failures. Aliment. Pharmacol. Ther. doi:10.1111/j.1365-2036.2012.05053.x 